\title{
Stability Analysis of a Worm Propagation Model with Time Delay and Quarantine
}

\author{
Tao $\mathrm{Li}^{1, \mathrm{a}^{*}}$,Yuanmei Wang ${ }^{1, \mathrm{~b}}$, Xiongding Liu ${ }^{1, \mathrm{c}}$ and Xisheng Zhan ${ }^{2, \mathrm{~d}}$ \\ ${ }^{1}$ School of Electronics and Information, Yangtze University, Jingzhou, Hubei, 434023, P.R. China \\ ${ }^{2}$ College of Mechatronics and Control Engineering, Hubei Normal University, Huangshi, \\ 435002, P. R. China

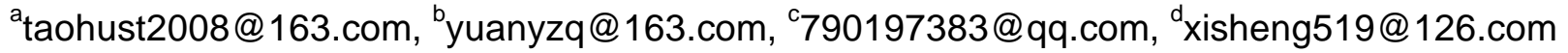

Keywords: Worm, Propagation Model, Time Delay, Quarantine, Stability

Abstract. There has a constant barrage of worms over the internet in recent years. In order to effectively prevent worm spreading, researchers are trying to understand the behavior of the worm propagation with the aid of epidemiological models. In this paper a worm propagation model with time delay and quarantine strategy is proposed and discussed. The spreading dynamics of the worms is analyzed, the spreading critical threshold and equilibriums are derived. The global stability of equilibriums is proved and numerical simulations are presented to confirmed the analytical results.

\section{Introduction}

Computer worms are an increasing problem throughout the world. Staniford et al. describe a worm that could infect the entire Internet in about 30s [1]. Researchers are trying to understand the behavior of the worm propagation with the aid of epidemiological models[2-11]. Both traditional and network-based epidemiological models have been applied to computer contagion. The traditional epidemiological model is Kermack-Mckendrick model. There are four main illustrative networks, each of which is vulnerable to attack: (A) a network of possible connections between computers using the Internet Protocol (IP), (B) a network of e-mail messages passed between users, (C) a network of e-mail address books, and (D) a network of shared administrator accounts for desktop computers. In network A, we consider nodes are IP addresses and two nodes are connected if communication is possible between the corresponding computers. So all nodes have the same degree in network A

In this paper, we mainly study the dynamic behavior of epidemic in network A. The remainder of the paper is organized as follows. Section 2 gives a brief review of the classical Kermack-Mckendrick model. Section 3 presents an extended worm spreading model, and the critical threshold value and equilibriums are derived. In section 4, we study the globally asymptotical stability of the equilibriums. In Section 5, the numerical simulations are provided. At last a brief conclusion is given in Section 6.

\section{Classical General Epidemic Model: Kermack-Mckendrick Model}

Kermack-Mckendrick model is also called the classical SIR epidemic model. Nodes that are vulnerable to be infected are called susceptible nodes, nodes that have been infected called infectious nodes, nodes that are immune or dead are called removed nodes. The density of susceptible, infectious and removed nodes is denoted by $S(t), I(t)$ and $R(t)$ separately at time $t$. The SIR model is

$$
\left\{\begin{array}{l}
\frac{d S}{d t}=-\beta I S \\
\frac{d I}{d t}=\beta I S-\alpha I \\
\frac{d R}{d t}=\alpha I
\end{array}\right.
$$

where $\beta$ is the infection rate; $\alpha$ is the rate of removal. 


\section{An Extension for the SIR model with Time Delay and Quarantine strategy}

In order to reduce transmissions of infectious nodes one efficient measure is to isolate infectious nodes. So, we introduce a class $Q$ of quarantined nodes. Considering the loss of immunity, the removed nodes $R(t)$ join the susceptible nodes $S(t+\tau)$ at time $t+\tau$ again. Using the same notation as the SIR model we obtain a differential system with time delay and quarantine strategy:

$$
\left\{\begin{array}{l}
\frac{d S}{d t}=L-\beta S I-d S+\varepsilon Q(t-\tau) e^{-d \tau} \\
\frac{d I}{d t}=\beta I S-d I-\delta I \\
\frac{d Q}{d t}=\delta I-d Q-\varepsilon Q \\
\frac{d R}{d t}=\varepsilon Q-d R-\varepsilon Q(t-\tau) e^{-d \tau}
\end{array}\right.
$$

Where $L$ is increase rate, $d$ is the decrease rate, $\delta$ is the quarantinable rate, $\varepsilon$ is the loss rate of immunity, the other parameters are the same as the SIR model in the previous sections. At the same time we assume that the new nodes are all susceptible. The total nodes $\operatorname{size} N$ satisfies $N^{\prime}=A-d N$. So the feasible region of system (1) is $M=\left\{(S, I, Q, R) \in R_{+}^{4} \mid S+I+Q+R \leq A / d\right\}$, and $M$ is the positively invariant region. Let $\rho=\frac{\beta L}{d(d+\delta)}$. System (1) has possibly two equilibriums: the disease-free equilibrium $E_{0}(L / d, 0,0,0)$ and the positive equilibrium $E^{*}\left(S^{*}, I^{*}, Q^{*}, R^{*}\right)$, where $S^{*}=\frac{L}{d \rho}, I^{*}=\frac{L(d+\varepsilon)(1-1 / \rho)}{(d+\delta)(d+\varepsilon)-\varepsilon \delta e^{-d \tau}}, Q^{*}=\frac{L \delta(1-1 / \rho)}{(d+\delta)(d+\varepsilon)-\varepsilon \delta e^{-d \tau}}, R^{*}=\frac{L \varepsilon \delta(1-1 / \rho)\left(1-e^{-d \tau}\right)}{d\left[(d+\delta)(d+\varepsilon)-\varepsilon \delta e^{-d \tau}\right]}$. Obviously, we obtain theorem 1.

Theorem 1. There always exists a disease-free equilibrium $E_{0}$ and when $\rho>1$, system (1) has a positive equilibrium $E^{*}$.

\section{The Stability of Equilibriums}

Theorem 2. $E_{0}$ is globally asymptotically stable for $\rho \leq 1$ and is unstable for $\rho>1$.

Proof. The Jacobian matrix at $E_{0}$ is

$$
J=\left[\begin{array}{cccc}
-\beta I^{0}-d & -\beta S^{0} & \varepsilon e^{-d \tau} & 0 \\
\beta I^{0} & \beta S^{0}-d-\delta & 0 & 0 \\
0 & \delta & -d-\varepsilon & 0 \\
0 & \alpha & \varepsilon\left(1-e^{-d \tau}\right) & -d
\end{array}\right]
$$

Obviously, If $\rho \leq 1 E_{0}$ is locally asymptotically stable, If $\rho>1 E_{0}$ is unstable. For $\rho \leq 1$, using the Lyapunov function $V=I$ with the Lyapunov derivative along the solutions of system (1), we get

$$
V^{\prime}=\beta I S-d I-\delta I \leq[\beta L / d-(d+\delta)] I \leq 0
$$

By the limiting equation theory and the Lyapunov-LaSalle invariance principle, $E_{0}$ is globally asymptotically stable. This completes the proof.

Theorem 3. If $\rho>1, E^{*}$ is globally asymptotically stable in the region $M-\{(S, I, Q, R) \mid I=0\}$. 
Proof. The Jacobian matrix at $E^{*}$ is

$$
J=\left[\begin{array}{cccc}
-\beta I^{*}-d & -\beta S^{*} & 0 & 0 \\
\beta I^{*} & \beta S^{*}-d-\alpha-\delta & 0 & 0 \\
0 & \delta & -d-\varepsilon & 0 \\
0 & \alpha & \varepsilon & -d
\end{array}\right] .
$$

Obviously, If $\rho>1 E^{*}$ is locally asymptotically stable. In order to prove the global asymptotic stability, consider the SI subsystem of system (1). The Lyapunov function

$$
V=S^{*} \int_{S^{*}}^{S} \frac{\beta u-(d+\delta)}{u} d u+\int_{I^{*}}^{I} \frac{\beta S^{*} u+d S^{*}-A}{u} d u
$$

is positive definite and goes to infinity as $S \rightarrow+\infty$ or $I \rightarrow+\infty$. Using $\beta S^{*}=d+\delta$, the $V$ derivative is

$$
\begin{aligned}
V^{\prime} & =S^{*} \beta\left(S-S^{*}\right)\left(L-\beta S I-d S+\varepsilon Q(t-\tau) e^{-d \tau}\right) / S+\beta\left(\beta S^{*} I+d S^{*}-L-\varepsilon Q(t-\tau) e^{-d \tau}\right)\left(S-S^{*}\right) \\
& \leq-\beta\left(L+\varepsilon Q(t-\tau) e^{-d \tau}\right)\left(S-S^{*}\right)^{2} / S \leq 0
\end{aligned}
$$

Note that $V^{\prime}=0$ on the set where $S=S^{*}$. The largest positively invariant subset is the equilibrium $\left\{\left(S^{*}, I^{*}\right)\right\}$, so that the Lyapunov-Lasalle theorem[12] implies that $\left(S^{*}, I^{*}\right)$ is globally stable in $R_{+}^{2}$ and $\lim _{t \rightarrow+\infty} I(t)=I^{*}$. From the third equation in system (1) we obtain

$$
Q(t)=\left\{Q_{0}+\int_{t_{0}}^{t} \delta I(\tau) \exp \left[(d+\varepsilon)\left(\tau-t_{0}\right)\right] d \tau\right\} \exp \left[-(d+\varepsilon)\left(t-t_{0}\right)\right]
$$

By L'Hospital's rule we get $\lim _{t \rightarrow+\infty} Q(t)=\lim _{t \rightarrow+\infty} \frac{\delta I(t)}{\varepsilon+d}=\frac{\delta I^{*}(t)}{\varepsilon+d}=Q^{*}$. Similarly, $\lim _{t \rightarrow+\infty} R(t)=\lim _{t \rightarrow+\infty} \frac{\varepsilon \delta\left(1-e^{-l \tau}\right) I^{*}}{d}=R^{*}$. Form the above analysis, $E^{*}$ is globally attractive. Considering the locally asymptotical stability of $E^{*}$, $E^{*}$ is globally asymptotically stable in the region $M-\{(S, I, Q, R) \mid I=0\}$. This completes the proof.

\section{Numerical Simulations}

The sum of the infectious nodes and quarantined nodes, i.e. the whole infected nodes is regarded as a coordinate quantity, so use $S, I+Q$ and $R$ to construct coordinate system. The general system is configured as $L=3, d=0.001, \beta=0.0001, \varepsilon=0.05$. Let the initial value be $(800,100,0,0)$.

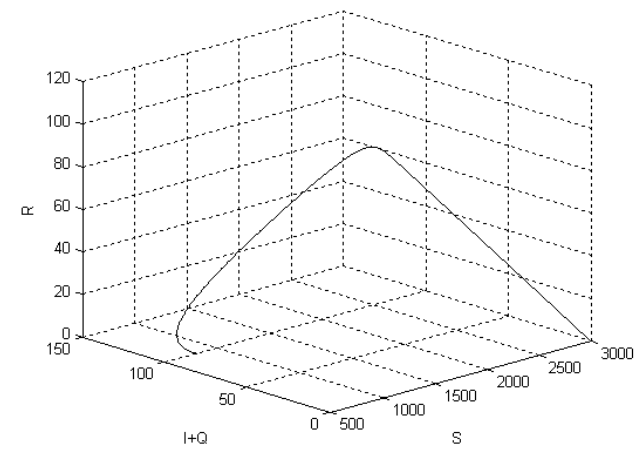

Fig.1. The trajectory of system (1) at $\delta_{1}=0.4$

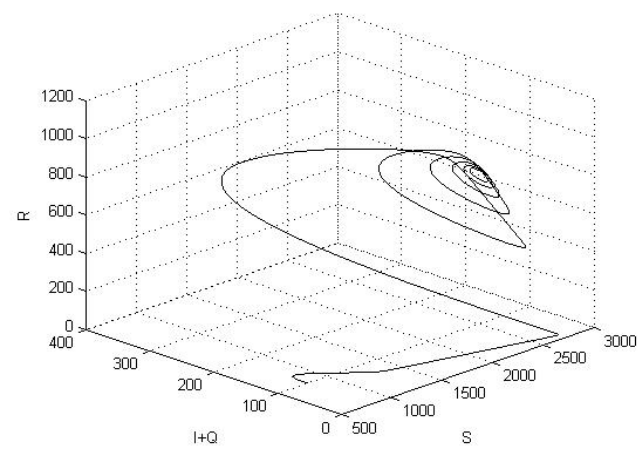

Fig.2. The trajectory of system (1) at $\delta_{2}=0.2$ 
We assume time delay $\tau=0$ in Fig.1 and Fig.2. From Theorem 2 we can get the critical value of quarantinable rate $\delta_{0}=0.299$. Fig. 1 shows that when $\delta_{1}=0.4$, which is larger than $\delta_{0}$, the trajectory of system (1) tends to the disease-free equilibrium $E_{0}(3000,0,0,0)$. Fig.2 shows that when $\delta_{2}=0.2$, which is less than $\delta_{0}$, the trajectory of system (1) tends to the positive equilibrium $E^{*}(1987,9,16,988)$.

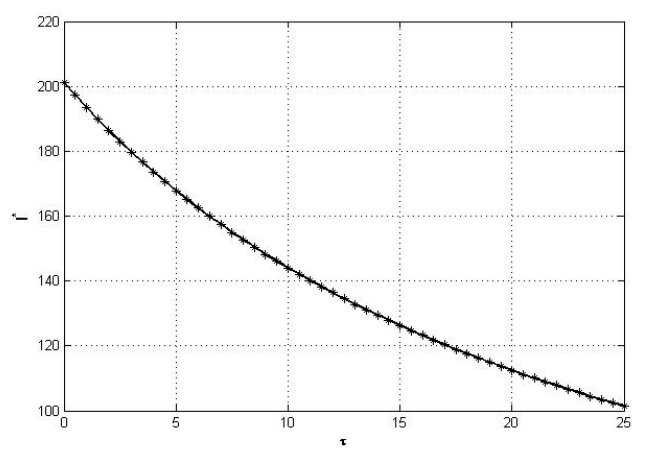

Fig.3. The relations between $I^{*}$ and $\tau$

We assume quarantine rate $\delta=0.2$ in Fig.3. Fig. 3 shows that when time delay $\tau$ increases the corresponding steady infection density $I^{*}$ decreases.

\section{Conclusions}

In this paper an extended worm propagation model with time-delay and quarantine strategy is presented. We have analyzed and obtained the existence conditions of the spreading threshold and equilibriums. The globally asymptotical stability of equilibriums and sufficient conditions are studied. Theoretical analysis and computer simulations have shown that time delay and quarantine strategy can decrease the steady infection density of the systems. The study has valuable guiding significance in effectively preventing worm spreading.

Acknowledgments. This work is partially supported by the National Natural Science Foundation of China under Grants 61471163.

\section{References}

[1] S. Staniford, V. Paxson, and N. Weaver, How to Own the Internet in Your Spare Time, Proceedings of the 11th USENIX Security Symposium, 149-167, 2002.

[2] G. Zhu, X. Fu, G.Chen, Commun. Nonlinear Sci. Numer. Simul. 17 (2012) 2588-2594.

[3] Yoichi Enatsu, Yukihiko Nakata, Yoshiaki Muroya, Acta Math. Sci. 32 (2012) 851-865.

[4] J. Lou, T. Ruggeri, J. Math. Anal. Appl. 365 (2010) 210-219.

[5] S. Eubank, H. Guclu, Nature. 429 (2004) 180-184.

[6] L. Wang, G. Dai, SIAM J. Appl. Math. 68 (2008) 1495-1502.

[7] R. Pastor-Satorras, A. Vespingani, Phys. Rev. Lett. 63 (2001) 066177.

[8] L. Wang, G. Dai, SIAM J. Appl. Math. 68 (2008) 1495-1502.

[9] M. Barthelemya, A. Barratb, J. Theor. Biol. 235 (2005) 275-288.

[10] R. Pastor-Satorras, A. Vespingani, Phys. Rev. E 65 (2002) 035108.

[11] A. Donofrio, Nonlinear Anal.: Real Word Appl. 9 (2008) 1567-1572.

[12] J.K. Hale, Ordinary Differential Equations, 2nd Ed., Krieger, Basel, 1980. 